\title{
Religious Muslim American Mothers' Perceptions of Child Behavior Problems
}

\section{Naadia M. Chowdhury, David S. Glenwick, and Mark E. Mattson}

Fordham University

Naadia M. Chowdhury is now at School of Law, New York University.

The authors express their appreciation to the Muslim Student Associations of Columbia University, Fordham University, and Hunter College and to the Jamaica Muslim Center for their facilitation of this research.

Correspondence concerning this article should be addressed to David S. Glenwick, Department of Psychology, Fordham University, Bronx, NY 10458. Email: glenwick@fordham.edu

\section{Abstract}

The present study investigated Muslim American mothers' perceptions of and reactive actions to child behavior problems, as well as factors (type of problem, severity of problem, religiosity, acculturation, and child gender) associated with their perceptions and reactive actions. Twenty Muslim American mothers having a child between the ages of 4 and 18 responded to four vignettes created by crossing the two dimensions of problem type (internalizing and externalizing) and problem severity (low and moderate), as well as to demographic, religiosity, and acculturation measures. Type and severity of behavior problem had significant relationships with mothers' perceptions of seriousness, level of worriedness, and unusualness. Religiosity and acculturation were not associated with mothers' perceptions but were associated with some reactive actions. Also, the participants tended to turn for help to nonprofessional (e.g., family and friends) sources more than to fellow community members and medical/mental health professionals. Implications of the results for community-based education and intervention programs are considered. 
Mental health care services for children in need are highly underutilized, with most (as many as 90\%, depending upon the particular survey) not having their needs for professional services adequately met (Costello, Egger, \& Angold, 2005). To investigate reasons for this gap, researchers have focused on, among other aspects, parents and factors influencing parental problem perception and action. Parents are considered gatekeepers to mental health care services for children because they typically are the ones who determine what behaviors qualify as problematic. This, in turn, informs their reactions to these behaviors, including accessing mental health services or not (Ford, Hamilton, Meltzer, \& Goodman, 2008; Poduska, 2000).

Among the factors found to be associated with parental perceptions are type and severity of symptoms, adult's tolerance level, and financial burden (e.g., Hankinson, 2009; Sayal, 2004; Weisz et al., 1988). For example, Sayal (2004), in a 4-year longitudinal investigation of 1,168 children with hyperactivity, found symptom severity to strongly predict parental seeking of psychiatric services. Similarly, in a study (Hankinson, 2009) of 223 parents, parents regarded externalizing behaviors and comorbid (i.e., co-existing externalizing and internalizing) behaviors as more problematic than internalizing ones. Mothers were more likely to seek out professional services than were fathers. Additionally, low tolerance, high distress, knowledge of child psychopathology, and parental socioeconomic status were predictive of problem perception and help-seeking behaviors.

Similar findings regarding severity of symptoms have been reported in several cross- cultural studies. For instance, Weisz et al. (1988) found that both U.S. and Thai adults reported externalizing behaviors to be more distressing than internalizing behaviors. Employing vignettes describing children's fighting and fearfulness behaviors, Lambert et al. (1992) found that both U.S. and Jamaican adults had less tolerance for externalizing (i.e., fighting) behaviors than for internalizing (i.e., fearfulness) behaviors.

Religion and religiosity (i.e., the adherence to the beliefs and practices of a formal religion; Harari, Glenwick, \& Cecero, 2014) have been unstudied with respect to their potential relationships to parental perceptions of child behavior problems. The bulk of extant research involving religiosity has focused on professing Christians' views of the causes of and treatments for adult psychopathology. For example, in a study of 126 adult Protestants in Australia, Hartog and Gow (2005) found a positive association between religiosity and religious causal attributions (e.g., demonic possession) of major depression and schizophrenia. With respect to help-seeking behaviors, in a U.S. Christian college student sample $(N=235)$, higher religiosity was related to a greater tendency to seek out religious figures rather than professionals (Crosby \& Bossley, 2012). Similarly, Gass (1984), in a study involving 204 U.S. college students, found that Orthodox Christians (i.e., those adhering to traditional Christian 
beliefs, such as Christ's divinity) were more desirous that counselors be of the same religious background as themselves, compared to nonorthodox and nonChristian students.

With respect to religion/religiosity and mental health care, Muslim Americans have been studied relatively infrequently. However, a recent body of literature has begun to explore how Muslim Americans conceptualize and deal with mental illness. Aloud and Rathur (2009), studying factors that influence Arab American Muslims' attitudes about mental health care, surveyed 281 Muslim college students and community residents in Columbus, Ohio. They found that knowledge of mental health care, Muslim beliefs, and social stigma against seeking professional care were factors related to help-seeking behavior. The participants were also found to seek informal services (i.e., family and friends) before contacting formal mental health care services (i.e., psychologists and psychiatrists), perhaps a reflection of cultural norms. Imams have frequently been identified by Muslim Americans as nonprofessional, or informal, mental health care service providers (Abu-Ras, Gheith, \& Cournos, 2008; Padela, Killawi, Forman, DeMonner, \& Heisler, 2012). Abu-Ras et al. (2008), for example, in interviews of 102 Muslim Americans in $22 \mathrm{New}$ York City mosques, found that their respondents believed that mosques provided culturally competent and appropriate mental health care counseling, with imams' focus on religion and spirituality being important and necessary. Similarly, Padela et al. (2012)'s study of Michigan Muslim Americans in 13 focus groups reported that recitations of the Quran and praying were identified as important actions toward healing for both mental and physical illnesses. Agents of healing that were identified included God, imams, family, and health care providers, with God considered to be both the primary source of the illness and the most important figure for healing. Thus, studies to date on Muslim Americans indicate the strong contribution of religious belief and action to perceptions of and actions regarding mental illness. However, the participants often have been recruited from mosques, which may have pulled for a relatively high level of religiosity that is not generalizable to the overall Muslim American population. Also, none of the studies to date have looked at perceptions of child behavior.

The present study aimed to extend both the parental perception and the Muslim American mental health care literature by examining Muslim American mothers' perceptions of child behavior problems. Based on previous research (e.g., Hankinson, 2009) that found mothers to be the parent more likely to seek help, the present study surveyed solely mothers. Due to the results of the above-mentioned studies, intensity of belief was the aspect of Muslim religion focused on. Also, parental acculturation and child gender were examined for their potential associations with perceptions. Four vignettes were created crossing the two dimensions of (a) internalizing and externalizing behaviors and (b) low and moderate severity (operationalized below). 
Based on the parental perception literature (with non-Muslim American parents) to date and the extant literature on Muslim Americans' perceptions of adult psychological disorders, it was hypothesized that the externalizing vignettes would be regarded as more concerning (i.e., serious, worrisome, and unusual) than internalizing ones and that the moderate-severity vignettes would be regarded as more concerning than the low-severity ones. It also was expected that religiosity and degree of acculturation would be related both to perceptions of concern and to preferred courses of action. Finally, it was hypothesized that the participants would choose to seek help from nonprofessional sources (e.g., the other parent, relatives, friends, or teachers) more than from professional sources (e.g., pediatricians, psychiatrists, or psychologists).

\section{Method}

\section{Participants}

Participants for the study were drawn from two groups. One consisted of mothers who were recruited through their children who were student members in Muslim Student Associations (MSAs) at three New York City colleges. MSAs are student-run clubs dedicated to the study of Islam and Islamic issues. The second subset of mothers was recruited through a youth group at a New York City-based Islamic cultural and religious center (IC). The youth group is an allfemale peer group for adolescents to discuss Islamic culture and study Islam. Inclusion criteria for the study were the mother's ability to speak basic English (as reported by their child) and having a child between the ages of 4 and 18 .

Fifty members of these organizations with mothers meeting inclusion criteria were given surveys through the MSAs and the IC. Of the 50 eligible mothers, 20 participated. The participant mothers' mean age was $43.05(S D=6.53)$. Fifteen self-identified as Asian American, two as Arab American, one as Caucasian, one as African American, and one as West Indian. All were married. The mean number of years lived in the United States was $19.82(S D=9.09)$. Sixty percent had a male child in the inclusion age range, and $40 \%$ a female child in that range. The mean age of the child was $12.20(S D=4.30)$.

\section{Instruments}

Demographic questionnaire. Data were collected on mothers' age, race/ethnicity, marital status, the age of the child closest to 8 years old (that being the age of the child in the vignettes), and the gender of the child.

Religiosity questionnaire. Intensity of religious belief was assessed using the Sahin- Francis Scale of Attitude toward Islam (SFS; Francis, Sahin, \& Al- 
Failakawi, 2008). The participants rated their thoughts on a 5-point Likert-type scale ranging from 1 (strongly agree) to 5 (strong disagree) for 17 items, such as "I find it inspiring to listen to the Quran" and "Allah/God means everything to me." Within the scale are three negative items (e.g., "I think going to the Mosque is a waste of my time") that are reversed scored. The scale has been shown to be a reliable and valid assessment of Islamic religiosity comparable to other established religious scales (Francis et al., 2008). Also, in a study of 381 Muslim adolescents in England, Francis et al. (2008) found a significant correlation between scores on the measure and the number of times the participants prayed. They reported an internal reliability of .90 . In the present study, the internal reliability (Cronbach's alpha) was similarly high at .91.

Acculturation proxy measure. Acculturation was assessed by participants' answers to the question, "How many years have you lived in the United States? (Please give the number, even if it's been your whole life)." This question has been used as a proxy for acculturation in similar studies (e.g., Williamson, Knox, Guerra, \& Williams, 2014) and was utilized in the current study in order to limit the time demand on the participants.

Vignettes. Vignettes and questions accompanying the vignettes were adopted from a study by Hankinson (2009) on parental problem perception. Four of Hankinson's six vignettes depicting an 8-year-old child were included in the present research. Two described internalizing behaviors at less severe and moderately severe levels, respectively, and two described externalizing behaviors at less severe and moderately severe levels, respectively. Illustratively, the low-severity internalizing vignette read:

Imagine that your 8-year-old son/daughter has been rather shy. He/she hasn't liked to play with other kids and would rather just be left alone. Recently, your child has been more self-conscious about what he/she says and does, especially in front of other peers. For instance, he/she doesn't want to wear certain clothes to school and doesn't want to try new things for fear of failure. $\mathrm{He} / \mathrm{she}$ also tends to be perfectionistic and has been getting upset when he/she messes up.

In comparison, the moderate-severity internalizing vignette described the child as "worrying a lot" and "crying more easily." The externalizing vignettes targeted argumentative and impulsive behaviors, with the moderate-severity vignette indicating more severe and frequent outbursts of those behaviors.

The vignettes were ordered as follows: low-severity internalizing, lowseverity externalizing, moderate-severity internalizing, and moderate-severity externalizing. It was believed that this order would be the least threatening to respondents. The less severe vignettes utilized behaviors from the Child Behavior Checklist (CBCL) that were prevalent in about 50\% and higher of 
nonreferred 8-to-9-year-old girls and boys in the CBCL's normative sample. The moderately severe vignettes utilized behaviors from the CBCL that were prevalent in $20 \%$ to $50 \%$ of the normative sample.

While reading each vignette, the mothers were asked to imagine their own child exhibiting the behaviors described in the vignette. Following each vignette, they were asked to answer the following four questions: "How serious is his/her behavior?" "How worried would you be about his/her behavior?" "Do you think his/her behavior would improve in a year or two?" and "Compared to other 8-year-olds, how unusual is his/her behavior?" on a 7-point Likerttype scale, ranging from 1 (not at all) to 7 (very much). They also were asked to select from a list of 11 actions what they would do regarding their child's behaviors in each vignette. The actions included doing nothing, or talking to the other parent, the child, a teacher, school counselor, friends and family, a religious leader, other community members, a physician, a psychologist, and a psychiatrist, respectively. Hankinson (2009) provided reliability and validity data in support of these vignettes.

\section{Procedure}

Following institutional review board approval, the participants were recruited through their offspring in the above-noted organizations. The principal investigator visited the four organizations during their general meetings in order to approach mothers' offspring for recruitment. Members of the organizations who had qualifying mothers were given a questionnaire and a stamped return envelope. When mothers received questionnaires from their children, they first read the informed consent form. Because of the anonymous nature of the surveys, the participants acknowledged informed consent when they mailed the questionnaire back to the principal investigator. The participants then completed the demographic form, followed by the SFS and the four vignettes. The demographic form and the SFS were positioned ahead of the vignettes because their content was likely familiar to the respondents, and therefore less threatening, than the vignettes. Upon completion of the questionnaire, the participants mailed it back to the principal investigator in the supplied envelope. The mothers were asked to return the questionnaire within a week of receiving it.

\section{Statistical Analysis}

Analyses of variance (ANOVAs) were conducted to analyze whether the type and severity of child behavior problem were related to mothers' perceptions. Pearson product- moment correlations examined whether religiosity and acculturation were associated with mothers' perceptions. The relationship between child gender and mothers' perceptions was analyzed by $t$ tests. 
Chi-square analyses for each of the vignettes compared the observed and expected frequencies across the help-seeking categories to see if the mothers preferred various courses of action over others. Finally, the relationships between religiosity and courses of action and between acculturation and courses of action were investigated with $t$ tests.

\section{Results}

Descriptive Data and Preliminary Analyses

To justify pooling the data for the MSA and IC participants, independent samples $t$ tests and a chi-square test were run to explore possible between-group differences. There were no significant differences with respect to religiosity, mothers' age, and child gender. There was a significant difference between MSA acculturation scores, $M=22.87$ ( $S D=7.02)$, and IC acculturation scores, $M=$ $10.67(S D=8.95), t(18)=3.15, p=.005$, with the IC participants living in the United States for fewer years than the MSA participants. (A potential outlier was recognized in the IC group, with one participant having lived in the United States for only 4 months. The between-groups difference in length of time in the United States remained significant when that outlier was removed, $p=.007$. Also, all subsequent inferential analyses [see below] were conducted both with and without that participant's data and produced similar results. Therefore, the data from the outlier participant were retained.) Given the general absence of between-group differences, the data for the two groups were pooled.

The mean religiosity score was $78.90(S D=6.64)$, which is classified as highly religious by the creators of the SFS. Correlational analyses among maternal age, religiosity, and acculturation were all nonsignificant.

Table 1 presents the means and standard deviations for each of the four vignettes with respect to mothers perceptions of seriousness, worrisomeness, unusualness, and likelihood of improvement with a year or two. These will be discussed below in the inferential analyses.

\section{Inferential Analyses}

To analyze whether the type (internalizing or externalizing) and severity (low or moderate) of child behavior problem were related to mothers' perceptions, ANOVAs were conducted. There was a significant main effect for type with respect to how serious mothers perceived the behaviors to be, $F(1,19)=14.90$, $p=.001$, with the externalizing vignettes considered to be more serious than the internalizing vignettes. There was also a significant main effect for severity with respect to how serious mothers perceived the behaviors to be, $F(1,19)=$ 
Figure 1. Interaction effect between type and severity of behavior problem with respect to seriousness scores.

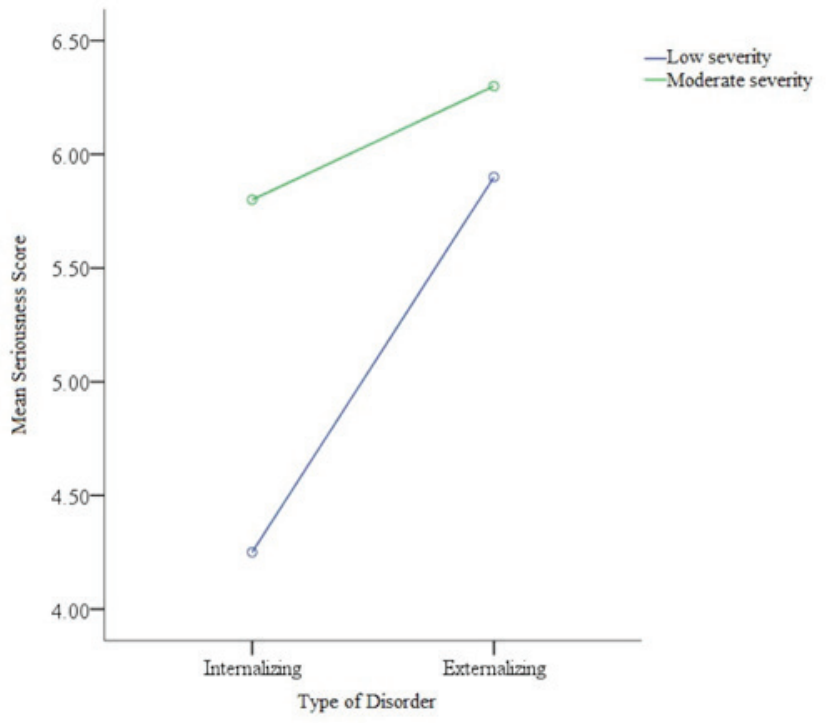

15.61, $p=.001$, with the moderate-severity vignettes considered to be more serious than the low- severity vignettes. There was also a significant interaction effect between type and severity, $F(1,19)=7.12, p=.015$, with the low severity internalizing vignette considered to be much less serious than the other three vignettes (see Figure 1).

There were significant main effects regarding how worried mothers felt about the child behaviors for both type, $F(1,19)=4.81, p=.041$, and severity $F(1,19)=7.91, p=.011$, of vignette. Mothers found the internalizing vignettes to be less worrisome than the externalizing vignettes, and the low-severity vignettes to be less worrisome than the moderate-severity vignettes. A significant interaction effect between type and severity was also found, $F(1,19)=4.53, p$ $=.047$, with the low-severity internalizing vignette being less worrisome than the other three (see Figure 2).

Lastly, a significant main effect was found for severity with respect to how unusual the mothers found the child behavior to be, $F(1,19)=8.58, p=.009$, with the moderate-severity vignettes being viewed as more unusual than the low-severity ones. There was no main effect for type and no interaction effect.

There were no main or interaction effects for type and severity with respect to likelihood of improvement within 1 to 2 years. The means for all four vignettes were in the likely-to- improve range (see Table 1).

Tests examining the correlations between religiosity and acculturation, on the one hand, and mothers' perceptions, on the other, were not significant. 
Figure 2. Interaction effect between type and severity of behavior problem with respect to worrisomeness scores.

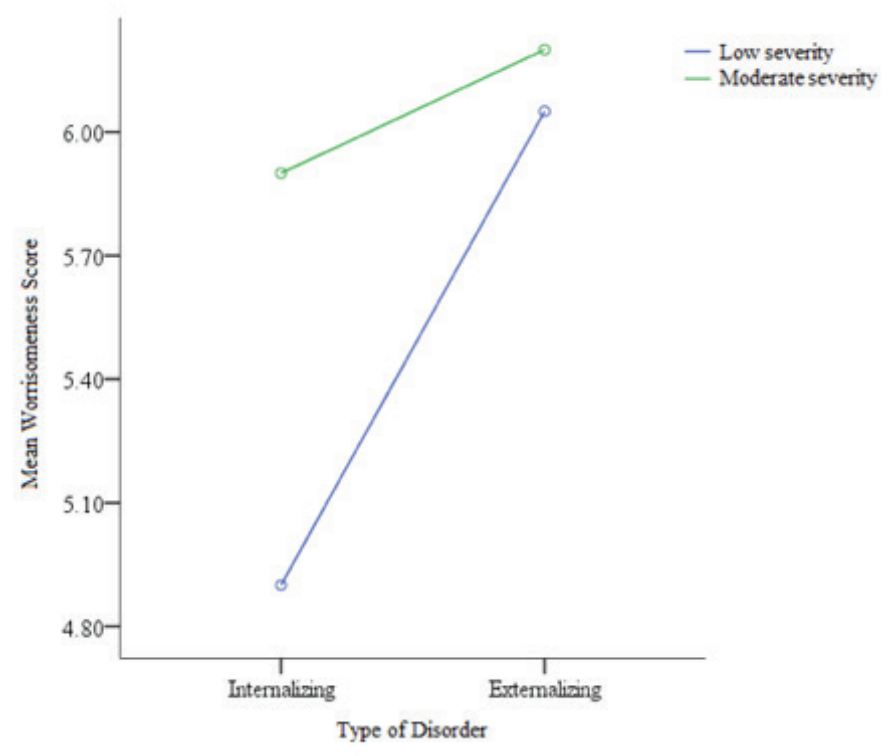

With respect to the relationship between child gender and mothers' perceptions, $t$ tests indicated that for the low-severity internalizing vignette, mothers reported a higher likelihood of improvement for females, $M=5.75(S D=.46)$, than for males, $M=4.75$ ( $S D=1.22$ ), $t(18)=2.21, p=.041$.

Table 2 presents frequency tables detailing the mothers' chosen courses of action. As can be seen, in only one instance (the low-severity internalizing vignette) did a mother say that she would do nothing. For statistical analysis purposes, the other possible choices were collapsed into five categories: direct social network (other parent, child, and family/friends); school-based resources (school counselor and teacher); imams; community members; and professional resources (physician, psychologist, and psychiatrist). Next, chi-square analyses were conducted for each of the four vignettes, comparing the observed and expected frequencies across the five categories. All four chi-squares were significant: $\chi 2(4, n=76)=29.11, p<.001$, for the low- severity internalizing vignette; $\chi 2(4, n=89)=44.75, p<.001$, for the low-severity externalizing vignette; $\chi 2(4, n=98)=29.22, p<.001$, for the moderate-severity internalizing vignette; $\chi 2(4, n=102)=38.02, p<.001$, for the moderate-severity externalizing vignette. The pattern of chosen actions was very consistent across the four vignettes. Specifically, direct social network members would be turned to at a greater than expected frequency, school-based resources and imams would be turned to at expected frequencies, and community members and professional 
TABLE 1. Means and Standard Deviations for Vignettes

\begin{tabular}{lcccccccc}
\hline Variable & \multicolumn{8}{c}{ Vignette } \\
\hline & $\begin{array}{c}\text { Low } \\
\text { internalizing }\end{array}$ & \multicolumn{2}{c}{$\begin{array}{c}\text { Moderate } \\
\text { internalizing }\end{array}$} & $\begin{array}{c}\text { Low } \\
\text { externalizing }\end{array}$ & \multicolumn{2}{c}{$\begin{array}{c}\text { Moderate } \\
\text { externalizing }\end{array}$} \\
\hline Serious & $M$ & $S D$ & $M$ & $S D$ & $M$ & $S D$ & $M$ & $S D$ \\
Worrisome & 4.25 & 1.62 & 5.90 & 1.29 & 5.80 & 1.06 & 6.30 & 1.17 \\
Likely to improve & 5.90 & 1.52 & 6.05 & 1.28 & 5.90 & 1.21 & 6.20 & 0.95 \\
Unusual & 5.15 & 1.09 & 4.95 & 1.10 & 4.95 & 1.39 & 4.90 & 1.48 \\
\hline
\end{tabular}

Note. $N=20$.

resources would be turned to at less than expected frequencies. The one exception was that for the moderate-severity externalizing vignette, school-based resources were chosen at a greater than expected frequency.

The relationships between religiosity and the various courses of action and between acculturation and the various courses of action were investigated with $t$ tests. A significant difference in religiosity was found for the low-severity internalizing vignette between mothers who would talk to other community members, $M=72.80$ ( $S D=7.05)$, and mothers who would not, $M=80.90$ (SD $=5.28), t(18)=2.75, p=.013$, with higher religiosity scores for those mothers who would not speak with other community members. For acculturation, there were significant differences between mothers who would talk with a religious leader, $M=11.27(S D=9.74)$, and mothers who would not, $M=22.67$ $(S D=7.08), t(18)=2.85, p=.011$, for the low- severity internalizing vignette and between mothers who would talk with a religious leader, $M=11.62$ (SD $=7.83)$, and mothers who would not, $M=24.23(S D=6.30), t(18)=3.93, p=$ .001 , for the low-severity externalizing vignette. For both of these low-severity vignettes, mothers who would speak with a religious leader had lived in the United States for a shorter period of time than those who would not.

\section{Discussion}

The present study extends the literature on Muslim American mental health by being the first to investigate parental perceptions. Also, it extends the literature on parental perception of child behavior issues by being the first to focus on Muslim American parents.

Tests investigating associations between type and severity of vignettes and mothers' perceptions indicated that both type and severity are related to how mothers' perceive child behavior issues. Moderate-severity behavior and externalizing type of behavior were perceived to be more problematic by Muslim 


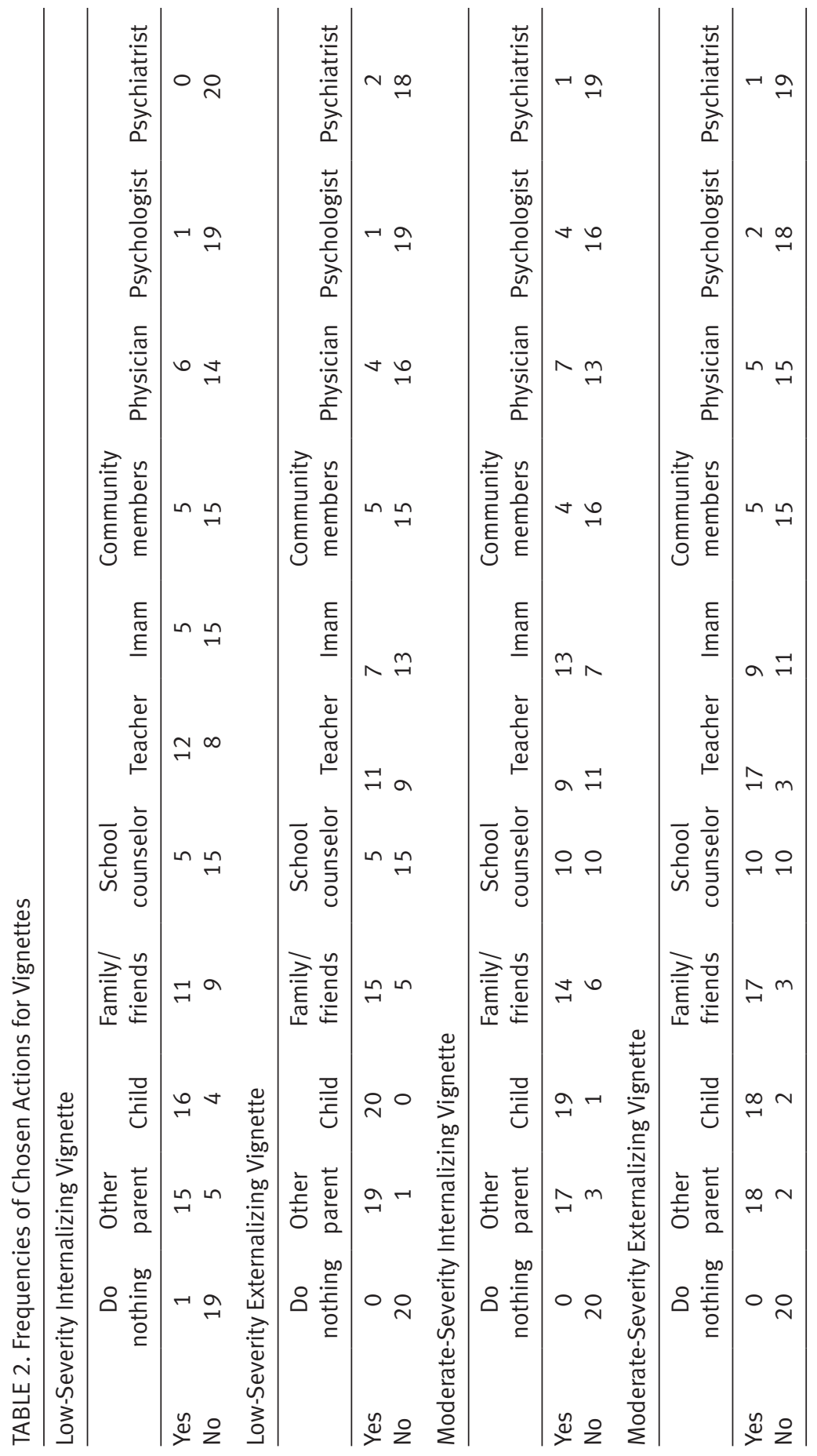


American mothers than were low-severity behavior and internalizing type of behavior, respectively. Additionally, the internalizing type combined with lowseverity - that is, the low-severity internalizing vignette-tended to be the least concerning of the four combinations, and much less so than the other three. The obtained differences with respect to type and severity of vignettes support the results of previous studies with other parent populations, both within and outside the United States (e.g., Hankinson, 2009; Lambert et al., 1992; Sayal, 2003; Weisz et al., 1988). These significant results add to the recognition that externalizing disorders are considered more problematic than internalizing disorders and moderate-severity disorders more problematic than low-severity disorders.

Courses of action chosen by the mothers indicated that they most preferred seeking help from close nonprofessional sources, that is, their direct social network. They also were fairly comfortable turning to school-based resources (especially teachers) or imams, but they were least likely to choose community members or professional medical/mental health resources. These findings, with our largely Asian American sample, is consistent with research on perceptions of adult psychological disorders with adult, largely Arab American, Muslim samples (Abu-Ras et al., 2008; Aloud \& Rathur, 2009; Padela et al., 2012). The infrequent desire to utilize community and professional sources of assistance may reflect the mothers' predictions of improvement. That is, regardless of type and severity of vignette, the mothers consistently regarded the behaviors as fairly transient (i.e., likely to improve within a year or two), rather than as longstanding psychological disorders or medical problems. These results may also indicate an unfamiliarity with possible professional sources and/or a mistrust of sources outside the circle of daily contact. With respect to the latter, potential social stigma and the risk of being judged negatively by one's peers may discourage parents from speaking to other community members about the behavior of one's child. Such an interpretation is consistent with Aloud and Rathur's (2009) finding that fear of social stigma was negatively related to help-seeking behavior in Muslim adults.

Religiosity and acculturation were more predictive of courses of action than of perceptions of problems. For perceptions, the effects of religiosity and acculturation were overridden by type and severity of behavior problem. It should be noted that the variability for religiosity scores was quite low, with all of the women scoring within the high-religiosity range. This lack of variability may have contributed to the nonsignificant results. The high religiosity scores may be reflective of the general Muslim population or might have be the result of recruiting from MSAs and an Islamic Center. Both types of places have members who are investing time in their religious beliefs and running organizations dedicated to Islam. This dedication may be a reflection of religious val- 
ues emphasized in the home by parents. The absence of associations between number of years living in the United States and perceptions of problems may be due to the former being only an imperfect approximation of acculturation (see discussion of limitations below).

Several significant findings demonstrated that religiosity and acculturation do have importance with respect to courses of action mothers would take for child behavior issues. Mothers who would seek out community members to speak with about their child's behavior issues had lower religiosity scores than mothers who would not. This finding is in line with Aloud and Rathur's (2009) report of a negative association between traditional Muslim beliefs and helpseeking behavior in adults. If religiosity is understood to describe a particular relationship and belief system with God, then it makes sense that mothers who were more religious refrained from sharing their problems with other people. It might be speculated that these women would engage in prayer as a means to seek out God to assist them before seeking others with whom to discuss the issue.

With respect to acculturation, the number of years that the mothers had lived in the United States was predictive of whether or not mothers chose to speak with a religious figure, an imam, for both of the low-severity vignettes. Parents living in the United States for a greater number of years are likely to be more aware of, and more comfortable turning to, resources beyond their immediate spiritual leader.

Analyses concerning the relationship of child gender to mothers' perceptions of vignette behavior were not significant, except for mothers' beliefs about the likelihood of behavior improvement for the low-severity internalizing vignette. The general absence of differences between male and female children support previous investigations involving this variable (Hankinson, 2009). The significant result for the low-severity internalizing vignette may be due to behavioral differences ascribed by parents to males and females. That is, females may be thought to be more likely to improve than males because internalizing behaviors overlap with behaviors, such as shyness, typically associated with girls. Males displaying internalizing behaviors may therefore be regarded as exhibiting more serious symptoms because young male children are assumed to be normatively outgoing and active (Hankinson, 2009).

Four limitations of this study should be noted. The first was the relatively small sample size. However, it should be noted that (a) the response rate (20 out of 50, for 40\%) was comparatively high for this type of research and (b) the sample had the advantage of being more community drawn, rather than having been obtained from populations associated with mosques, as most previous studies with Muslim Americans had been.

A second limitation was that the method of recruitment was indirect, with 
the questionnaires being handed to each potential participant's adolescent or young adult offspring rather than to the parent herself. Despite the specific instructions that were provided, it is not known how this indirect approach may have influenced the transmission and completion of the questionnaires. Given that Muslim American mothers are likely fairly private and not comfortable with an outsider surveying them-an observation made by one of the IC's administrators (S. Khan, personal communication, February 5, 2014), researchers might benefit from spending time at the beginning of a study cultivating rapport with the community in order to develop a trusting relationship and more directly access potential participants.

A third limitation involves the religiosity and acculturation measures employed. The Sahin-Francis Scale of Attitude toward Islam inquires into respondents' religious beliefs (e.g., "I feel that I am very close to Allah/God"), rather than religious practices (e.g. asking participants how many times they pray in a day). Assessing both beliefs and practices would potentially provide a more complete understanding of the participants' religiosity and result in greater variability in religiosity. Acculturation, as previously mentioned, was assessed by the number of years lived in the United States. Although often employed as a brief acculturation measure, this item is only a rough approximation of acculturation and might not have adequately measured how well the participants had adapted into American culture. This is especially true for a city, like New York, having many ethnic enclaves. It is possible for an individual to reside within such enclaves for a number of years without acculturations into the larger society. Therefore, utilization of a more in-depth scale of acculturation (e.g., with respect to customs and languages) could result in more valid measurement of this construct.

A final limitation involves sample bias. The particular organizations sampled resulted in a sample that was largely Asian American. Thus, the results might not be reflective of other Muslim Americans, such as Arab Americans, African Americans, and nonbelieving Muslims.

With respect to future research, studies of Muslim American parental perception could look into the potential roles of additional factors, such as financial resources and knowledge of psychopathology, that have been found to be predictive in other populations. Additionally, the present questionnaire approach would profit from being complemented by qualitative methodology investigating mothers' conceptualization of child behavior problems (e.g., regarding biological, psychological, and/or social causality).

As the findings indicated that the mothers preferred nonprofessional resources that were part of their social networks, intervention programs could target increasing the use of such community resources. For example, programs creating support groups in community organizations and/or promoting utilization of already existing support mechanisms could be beneficial in address- 
ing unmet needs. Relatedly, as imams often were turned to for advice, providing them with mental health and counseling training could supplement their competencies and perceived virtues. Finally, educating communities regarding psychopathology and clinical services could provide Muslim American parents with knowledge of resources concerning child behavior problems.

The present study represents a first step in exploring Muslim American parents' perceptions of child behavior issues. Hopefully, future research will attempt to build upon the current findings to both add to the empirical literature and contribute to the development of effective educational and therapeutic programs.

\section{References}

Abu-Ras, W., Gheith, A., \& Cournos, F. (2008). The imam's role in mental health promotion: A study at 22 mosques in New York City's Muslim community. Journal of Muslim Mental Health, 3, 155-176. doi.org/10.1080/15564900802487576

Aloud, N., \& Rathur, A. (2009). Factors affecting attitudes toward seeking and using formal mental health and psychological services among Arab Muslim populations. Journal of Muslim Mental Health, 4, 79-103. doi.org/10.1080/15564900802487675

Costello, E. J., Egger, H., \& Angold, A. (2005). 10-year research update reviews: The epidemiology of child and adolescent psychiatric disorders: I. Methods and public health burden. Journal of the American Academy of Child \& Adolescent Psychiatry, 44, 972-986. doi.org/10.1097/01.chi.0000172552.41596.6f

Crosby, J. W., \& Bossley, N. (2012). The religiosity gap: Preferences for seeking help from religious advisors. Mental Health, Religion \& Culture, 15, 141-159. doi.org/1 $0.1080 / 13674676.2011 .561485$

Ford, T., Hamilton, H., Meltzer, H., \& Goodman, R. (2008). Predictors of service use for mental health problems among British schoolchildren. Child and Adolescent Mental Health, 13, 32-40. doi.org/10.1111/j.1475-3588.2007.00449

Francis, L. J., Sahin, A., \& Al-Failakawi, F. (2008). Psychometric properties of two Islamic measures among young adults in Kuwait: The Sahin-Francis Scale of Attitude toward Islam and the Sahin Index of Islamic Moral Values. Journal of Muslim Mental Health, 3, 9-24. doi.org/10.1080/15564900802035201

Gass, C. S. (1984). Orthodox Christian values related to psychotherapy and mental health. Journal of Psychology and Theology, 12, 230-237.

Hankinson, J. C. (2009). Child psychopathology, parental problem perception, and helpseeking behaviors. Retrieved from ProQuest Digital Dissertations. (749943818).

Harari, E., Glenwick, D. S., \& Cecero, J. J. (2014). The relationship between religiosity/ spirituality and well-being in gay and heterosexual Orthodox Jews. Mental Health, Religion \& Culture, 17, 886-897. doi.org/10.1080/13674676.2014.942840

Hartog, K., \& Gow, K. M. (2005). Religious attributions pertaining to the causes and cures of mental illness. Mental Health, Religion \& Culture, 8, 263-276. doi.org/10. 1080/13674670412331304339

Lambert, M., Weisz, J. R., Knight, F., Desrosiers, M., Overly, K., \& Thesiger, C. (1992). Jamaican and American adult perspectives on child psychopathology: Further ex- 
ploration of the threshold model. Journal of Consulting and Clinical Psychology, 60, 146-149. doi.org/10.1037/0022-006X.60.1.146

Padela, A. I., Killawi, A., Forman, J., DeMonner, S., \& Heisler, M. (2012). American Muslim perceptions of healing: Key agents in healing, and their roles. Qualitative Health Research, 22, 846-858. doi.org/10.1177/1049732312438969

Poduska, J. M. (2000). Parents' perceptions of their first graders' need for mental health and educational services. Journal of the American Academy of Child \& Adolescent Psychiatry, 39, 584-591. doi.org/10.1097/00004583-200005000-00012

Sayal, K. (2004). The role of parental burden in child mental health service use: Longitudinal study. Journal of the American Academy of Child \& Adolescent Psychiatry, 43, 1328-1333. doi.org/10.1097/01.chi.0000138353.83357

Weisz, J. R., Suwanlert, S., Chaiyasit, W., Weiss, B., Walter, B. R., \& Anderson, W. (1988). Thai and American perspectives on over- and undercontrolled child behavior problems: Exploring the threshold model among parents, teachers, and psychologists. Journal of Consulting and Clinical Psychology, 56, 601-609. doi. org/10.1037/0022-006X.56.4.601

Williamson, A. A., Knox, L., Guerra, N. G., \& Williams, K. R. (2014). A pilot randomized trial of community-based parent training for immigrant Latina mothers. American Journal of Community Psychology, 53. 47-59. doi.org/0.1007/s10464013-9612-4 